\title{
Pulse Pressure and Upstroke Time Are Useful Parameters for the Diagnosis of Peripheral Artery Disease in Patients With Normal Ankle Brachial Index
}

\author{
Shunsuke Kiuchia, b, Shinji Hisatake ${ }^{\mathrm{a}}$, Ippei Watanabe ${ }^{\mathrm{a}}$, Mikihito Toda ${ }^{\mathrm{a}}$, Takayuki Kabuki ${ }^{\mathrm{a}}$, \\ Takashi Oka ${ }^{\mathrm{a}}$, Shintaro Dobashi ${ }^{\mathrm{a}}$, Takanori Ikeda ${ }^{\mathrm{a}}$
}

\begin{abstract}
Background: Some peripheral artery disease (PAD) patients have normal ankle brachial index $(\mathrm{ABI})(0.9$ - 1.4), although $\mathrm{ABI}$ is a useful parameter for the diagnosis of PAD. We investigated whether other parameters of ABI report sheet are useful to detect these patients.

Methods: We initially enrolled 3,912 patients (7,824 limbs) who underwent ABI for the first time. Subjects who have normal ABI were divided into the PAD group $(n=136)$ and the non-PAD group $(n=$ 240) by lower extremity ultrasonography. We investigated blood pressures (BP) (systolic (SBP), diastolic (DBP), mean (mBP) and pulse pressure (PP)), heart rate, upstroke time (UT), and \%mean arterial pressure (\%MAP).
\end{abstract}

Results: SBP, mBP, PP, UT, and \%MAP in the PAD group were significantly higher. A multivariate analysis showed that $\mathrm{mBP}, \mathrm{DBP}$, PP, UT and \%MAP were independently associated with the presence of PAD (mBP: odds ratio (OR) 2.30, 95\% confidence interval (CI) $1.22-4.37, \mathrm{P}=0.010$; DBP: OR $0.52,95 \%$ CI $0.28-0.97$, $\mathrm{P}=0.039$; PP: OR $1.30,95 \%$ CI $0.69-2.46, \mathrm{P}=0.041 ; \mathrm{UT}: \mathrm{OR}$ $3.40,95 \%$ CI $2.03-5.83, \mathrm{P}<0.001$; \%MAP: OR $1.77,95 \%$ CI $1.05-2.98, \mathrm{P}=0.031$ ). Maximal area under the curve (AUC) of BPs for associating PAD was PP. The cut-off value of PP was 53.0 $\mathrm{mm} \mathrm{Hg}$ (sensitivity 0.500 , specificity 0.721 , AUC $0.628,95 \%$ CI $0.569-0.687)$.

Conclusions: The present study demonstrated that BPs are associated with PAD in patients with normal ABI. The measurement of BPs could provide additional information for the diagnosis of PAD.

Keywords: Ankle brachial index; Pulse pressure; Upstroke time; Peripheral artery disease

Manuscript accepted for publication October 27, 2016

aDepartment of Cardiovascular Medicine, Toho University Faculty of Medicine, Tokyo, Japan

${ }^{b}$ Corresponding Author: Shunsuke Kiuchi, Department of Cardiovascular Medicine, Toho University Faculty of Medicine, 6-11-1 Omorinishi, Ota-ku, Tokyo 143-8541, Japan. Email: syunnsuke@med.toho-u.ac.jp

doi: http://dx.doi.org/10.14740/cr508e

\section{Introduction}

Japanese society is facing a problem of "super-aging". Although arteriosclerotic disease typified by acute coronary syndrome (ACS) is the main cause of death, it has been reported that the onset age of ACS has also growing order [1]. Moreover, the relationship between vascular age and arteriosclerotic diseases has also been reported [2]. Therefore, the prevention and treatment of these arteriosclerotic diseases are important. Ischemic heart disease (IHD), peripheral artery disease (PAD), cerebral vascular disease (CVD), and carotid artery stenosis are known as arteriosclerotic diseases, and these overlap as poly vascular disease (PVD). In the REduction of Atherothrombosis for Continued Health (REACH) registry, $19.0 \%$ of all patients were re-admitted for arteriosclerotic disease [3], the rate rose to $23.0 \%$ in patients with concomitant IHD and $33.6 \%$ in patients with concomitant PAD, and detection of PAD was the most useful criterion for evaluation of PVD. It has also been reported that the mortality rate increases in PAD patients compared with that in nonPAD patients regardless of the presence or absence of lower limb symptoms [4], showing the importance of diagnosing PAD.

Standard examination of the diagnosis for PAD is ankle brachial index (ABI) [5]. Guidelines for management of PAD by the Japanese Circulation Society show that the cut-off level of ABI is $0.9-1.4$. However, a value of $0.9-1.4$ is not necessarily normal. Therefore, the PAD diagnosis algorithm prepared by the American Heart Association (AHA) in 2011 recommends a treadmill exercise $\mathrm{ABI}$ test to examine these patients [6]. However, it is difficult to perform an exercise $\mathrm{ABI}$ test in all of these patients. In the present study, we investigated whether parameters of the $\mathrm{ABI}$ report can be used to detect PAD in patients with normal ABI $(0.9-1.4)$, retrospectively.

\section{Materials and Methods}

All experiments were performed in accordance with the Declaration of Helsinki and were approved by the Toho University Omori Medical Center Ethical Committee (25193). The present study was the retrospective analysis study design. 
Table 1. Patient Characteristics Showing No Differences Between Both Groups

\begin{tabular}{llll}
\hline & PAD group $(\mathbf{n}=\mathbf{1 3 6})$ & Non-PAD group $(\mathbf{n}=\mathbf{2 4 0})$ & P value \\
\hline Age $($ years $)$ & $70.3 \pm 9.7$ & $70.4 \pm 11.7$ & 0.510 \\
Male/female & $100 / 36$ & $166 / 74$ & 0.174 \\
Height $(\mathrm{cm})$ & $161.5 \pm 9.0$ & $160.0 \pm 9.4$ & 0.069 \\
Weight $(\mathrm{kg})$ & $61.1 \pm 11.6$ & $61.4 \pm 12.2$ & 0.589 \\
BMI $\left(\mathrm{kg} / \mathrm{m}^{2}\right)$ & $23.3 \pm 3.7$ & $23.9 \pm 3.9$ & 0.918 \\
Lower limb symptoms $(\%)$ & $30.1 \pm 46.1$ & $24.6 \pm 43.1$ & 0.121 \\
\hline
\end{tabular}

BMI: body mass index. Continuous data are expressed as the mean \pm standard deviation. $\mathrm{P}$ values were determined using the Student's $t$-test.

\section{Study subjects}

We initially enrolled 3,912 consecutive patients $(7,824$ limbs $)$ who underwent ABI testing from January 2009 to July 2015 at Toho University Omori Medical Center. We analyzed subjects with normal $\mathrm{ABI}(0.9$ - 1.4) who underwent $\mathrm{ABI}$ testing for the first time and examined by lower extremity ultrasound. Finally, 376 limbs were investigated. Limbs were divided into the PAD group $(\mathrm{n}=136)$ and the non-PAD group $(\mathrm{n}=240)$ by lower extremity ultrasound.

\section{General findings and medications}

Age, gender, height and weight were investigated. Moreover, we calculated body mass index (BMI), using the following formula: $\mathrm{BMI}=$ weight $(\mathrm{kg}) /$ height $(\mathrm{m})^{2}$. We investigated the prevalence of coronary risk factors such as diabetes mellitus (DM) and the percentage of patients with maintenance hemodialysis. Hypertension (HT) was diagnosed with administration of antihypertensive medications or diagnostic criteria of the Guidelines for the Management of HT [7]. As well, dyslipidemia (DLP) and DM were also diagnosed with administration of glucose/lipid lowering medications or diagnostic criteria of the Guidelines for the Management of DM/DLP [8]. In addition, medications to treat HT, DLP, and DM were investigated. The presence or absence of lower limb symptoms such as claudication or leg pain was also evaluated.

\section{ABI}

ABI was measured according to the methods described previously, using a VaSera VS-1500E manufactured by Fukuda Denshi Company, Ltd (Tokyo, Japan) [9]. The ABI of subjects was measured in the morning after $12 \mathrm{~h}$ of fasting. Their electrocardiogram and heart sounds were monitored after the subjects had been lying comfortably in the dorsal position for at least $10 \mathrm{~min}$. Cuffs were applied to the bilateral upper arms and ankles. Pulse wave velocity was obtained by dividing vascular length by the time taken for the pulse wave to propagate from the aortic valve to the ankle. We investigated blood pressures (BPs) (systolic blood pressure (SBP), diastolic blood pressure (DBP), mean blood pressure (mBP) and pulse pressure (PP)), heart rate, upstroke time (UT), \%mean arterial pressure (\%MAP) and cardio ankle vascular index (CAVI) from ABI report sheet. BPs were used in the arm with higher BPs. We calculated the average of CAVI from right and left CAVI and used these averages.

\section{Lower extremity ultrasonography}

Lower extremity ultrasonography was performed with two specialists (a cardiologist and a peripheral vascular ultrasound technician). We investigated lower extremity ultrasonography in subjects which had leg symptoms or some atherosclerotic risk factors. Peripheral artery stenosis is classified into four categories evaluated with lower extremity ultrasonography: 1) 0-39.9\% stenosis, 2) $40-69.9 \%$ stenosis, 3) $70-99.9 \%$ stenosis, and 4) completely occluded [10]. Categories 3 or 4 of the above criteria was defined as PAD.

\section{Statistical analysis}

Continuous variables were expressed as mean \pm standard deviation. We compared two groups by unpaired Student's $t$-test. Statistical significance was considered as $\mathrm{P}<0.05$ in all instances. First, univariate analysis was performed by applying Cox proportional hazard models for continuous variables. Second, factors found to be significant upon univariate analysis were included in a multivariate analysis. The receiver operating characteristic (ROC) curve was analyzed to determine an appropriate cut-off of parameters to predict for PAD. We used a Windows computer (Excel (Microsoft XP)) and EZR (Saitama Medical Center, Jichi Medical University), which is a graphical user interface for $\mathrm{R}$ (version 2.13.0, The R Foundation for Statistical Computing, Vienna, Austria) [11].

\section{Results}

\section{General findings and medications between both groups}

Mean age was $70.3 \pm 10.9$ years and 266 subjects were male. 
Table 2. Cardiovascular Risk Factors in Patients in the PAD Group

\begin{tabular}{|c|c|c|c|}
\hline & PAD group $(n=136)$ & Non-PAD group $(n=240)$ & P value \\
\hline Hypertension, n (\%) & $96(70.8)$ & $155(64.6)$ & 0.118 \\
\hline Dyslipidemia, n (\%) & $55(40.4)$ & $80(33.3)$ & 0.084 \\
\hline Diabetes mellitus, n (\%) & $49(36.0)$ & $49(20.4)$ & $<0.001$ \\
\hline Hemodialysis, $\mathrm{n}(\%)$ & $17(12.5)$ & $19(7.9)$ & 0.074 \\
\hline RAS-I, n (\%) & $42(30.9)$ & $63(26.3)$ & 0.169 \\
\hline Calcium channel blocker, n (\%) & $59(43.4)$ & $78(32.5)$ & 0.018 \\
\hline$\beta$-blocker, n (\%) & $42(30.9)$ & $63(26.3)$ & 0.169 \\
\hline Antihypertensive medications, n (\%) & $90(66.2)$ & $150(62.5)$ & 0.239 \\
\hline Number of antihypertensive medications & $1.48 \pm 1.37$ & $1.24 \pm 1.27$ & 0.043 \\
\hline HMG-CoA inhibitor, n (\%) & $49(36.0)$ & $59(24.6)$ & 0.009 \\
\hline Lipid lowering medications, n (\%) & $52(38.2)$ & $72(30.0)$ & 0.052 \\
\hline Glucose lowering medications, n (\%) & $45(33.1)$ & $46(19.2)$ & 0.001 \\
\hline
\end{tabular}

RAS-I: renin-angiotensin-aldosterone system inhibitor; HMG-CoA inhibitor: 3-hydroxy-3-methylglutaryl-coenzyme A inhibitor. Continuous data are expressed as the mean \pm standard deviation. $P$ values were determined using the Student's $t$-test.

General findings showed no differences between the two groups (Table 1). There was also no difference in the percentage of lower limb symptoms in the PAD group and non-PAD group. However, the percentage of PAD in subjects with lower limb symptoms was significantly higher compared with absence of lower limb symptoms $(41.0 \pm 49.4$ vs. $21.4 \pm 41.1$, $\mathrm{P}<0.001)$. Medical history and medications of study subjects were shown in Table 2. There was no significant difference, although the prevalence of HT and DLP was tended to be higher in the PAD group. The prevalence of DM in the PAD group was significantly higher than those in the non-PAD group. The percentage of patients with maintenance hemodialysis in the PAD group was tended to be higher, compared with the non-PAD group. No significant difference was noted in the rate of antihypertensive medication between the two groups, but that of the calcium channel blocker (CCB) was significantly higher in the PAD group. The number of antihypertensive medications was also significantly higher in the PAD group. No significant difference was noted in the rate of lipid lowering medications, but that of statin: HMG-CoA (3-hydroxy-3-methylglutaryl-coenzyme A) reductase inhibitor was significantly higher in the PAD group. The rate of glucose lowering medications was also significantly higher in the PAD group.

\section{ABI, CAVI and pulse wave between both groups}

These findings are shown in Table 3 . There was no significant difference in ABI between the two groups. CAVI in the PAD group was significantly higher than those in the non-PAD group $(9.40 \pm 1.99$ vs. $8.93 \pm 1.46, \mathrm{P}=0.005)$. Pulse waves were analyzed with \%MAP and UT, and these parameters in the PAD group were significantly higher than those in the nonPAD group (\%MAP: $44.5 \pm 21.7$ vs. $38.2 \pm 5.4 \mathrm{~mm} \mathrm{Hg}, \mathrm{P}<$ 0.001; UT: $170.7 \pm 45.9$ vs. $147.9 \pm 29.3 \mathrm{~ms}, \mathrm{P}<0.001)$.

\section{BPs between both groups}

SBP, $\mathrm{mBP}$ and PP in the PAD group were significantly higher than those in the non-PAD group (SBP: $144.2 \pm 21.5$ vs. 139.3 $\pm 20.0 \mathrm{~mm} \mathrm{Hg}, \mathrm{P}=0.014$; mBP: $109.8 \pm 19.6$ vs. $106.0 \pm 16.3$ $\mathrm{mm} \mathrm{Hg}, \mathrm{P}=0.024$; PP: $60.4 \pm 15.0$ vs. $54.0 \pm 13.1 \mathrm{~mm} \mathrm{Hg}, \mathrm{P}$ $<0.001$, Table 3 ). DBP was lower in the PAD group, but the difference was not significant (Table 3 ).

\section{A multivariate analysis for prediction of PAD}

Univariate analysis showed that ABI, CAVI, SBP, DBP, mBP,

Table 3. Blood Pressures in the PAD Group Were Higher Than Those in the Non-PAD Group

\begin{tabular}{llll} 
& $\begin{array}{l}\text { PAD group } \\
(\mathbf{n}=\mathbf{1 3 6})\end{array}$ & $\begin{array}{l}\text { Non-PAD group } \\
(\mathbf{n}=\mathbf{2 4 0})\end{array}$ & P value \\
\hline ABI & $1.03 \pm 0.08$ & $1.08 \pm 0.10$ & 1.000 \\
CAVI & $9.40 \pm 1.99$ & $8.93 \pm 1.46$ & 0.005 \\
\%MAP (\%) & $44.5 \pm 21.7$ & $38.2 \pm 5.4$ & $<0.001$ \\
UT (ms) & $170.7 \pm 45.9$ & $147.9 \pm 29.3$ & $<0.001$ \\
SBP (mm Hg) & $144.2 \pm 21.5$ & $139.3 \pm 20.0$ & 0.014 \\
DBP (mm Hg) & $83.8 \pm 13.2$ & $85.3 \pm 13.2$ & 0.863 \\
mBP (mm Hg) & $109.8 \pm 19.6$ & $106.0 \pm 16.3$ & 0.024 \\
PP (mm Hg) & $60.4 \pm 15.0$ & $54.0 \pm 13.1$ & $<0.001$ \\
Heart rate (bpm) & $70.2 \pm 13.4$ & $70.6 \pm 12.6$ & 0.597 \\
\hline
\end{tabular}

$\mathrm{ABI}$ : ankle brachial index; CAVI: cardio ankle vascular index; \%MAP: $\%$ mean arterial pressure; UT: upstroke time; SBP: systolic blood pressure; DBP: diastolic blood pressure; mBP: mean blood pressure; PP: pulse pressure. Continuous data were expressed as mean \pm standard deviation. $\mathrm{P}$ values were determined using the Student's $t$-test. 
Table 4. Univariate and Multivariate Analysis for Prediction of PAD

\begin{tabular}{|c|c|c|c|c|c|c|}
\hline & \multicolumn{3}{|c|}{ Univariate analysis } & \multicolumn{3}{|c|}{ Multivariate analysis } \\
\hline & HR & $95 \% \mathrm{CI}$ & $P$ value & HR & $95 \% \mathrm{CI}$ & $P$ value \\
\hline Age & 0.89 & $0.58-1.36$ & 0.586 & & & \\
\hline Men & 1.31 & $0.83-2.09$ & 0.250 & & & \\
\hline BMI & 0.88 & $0.56-1.37$ & 0.558 & & & \\
\hline $\mathrm{ABI}$ & 0.33 & $0.21-0.52$ & $<0.001$ & 0.49 & $0.30-0.81$ & 0.006 \\
\hline CAVI & 2.72 & $1.58-4.67$ & $<0.001$ & 1.64 & $0.89-3.03$ & 0.111 \\
\hline SBP & 1.01 & $1.00-1.02$ & 0.030 & 1.18 & $0.57-2.43$ & 0.660 \\
\hline DBP & 0.62 & $0.40-0.94$ & 0.026 & 0.52 & $0.28-0.97$ & 0.039 \\
\hline $\mathrm{mBP}$ & 1.93 & $1.16-3.21$ & 0.012 & 2.30 & $1.22-4.37$ & 0.010 \\
\hline PP & 1.03 & $1.02-1.05$ & $<0.001$ & 1.30 & $0.69-2.46$ & 0.041 \\
\hline Heart rate & 1.32 & $0.75-2.32$ & 0.335 & & & \\
\hline$\%$ MAP & 2.82 & $1.77-4.50$ & $<0.001$ & 1.77 & $1.05-2.98$ & 0.031 \\
\hline UT & 5.33 & $3.28-8.66$ & $<0.001$ & 3.44 & $2.03-5.83$ & $<0.001$ \\
\hline
\end{tabular}

BMI: body mass index; $\mathrm{ABI}$ : ankle brachial index; CAVI: cardio ankle vascular index; SBP: systolic blood pressure; DBP: diastolic blood pressure; mBP: mean blood pressure; PP: pulse pressure; \%MAP: \%mean arterial pressure; UT: upstroke time; HR: hazard ratio; $\mathrm{Cl}$ : confidence interval. Univariate analysis was performed by applying Cox proportional hazard models for continuous variables. Factors found to be significant upon univariate analysis were included in a multivariate analysis.

PP, \%MAP and UT were associated with the presence of PAD (Table 4). \%MAP and UT were independently associated with the presence of PAD in multivariate analysis (Table 4). In BPs, multivariate analysis indicated that DBP, mBP and PP were independently associated with the presence of PAD (DBP: odds ratio $(\mathrm{OR}) 0.52,95 \%$ confidence interval $(\mathrm{CI}) 0.28-0.97, \mathrm{P}=$

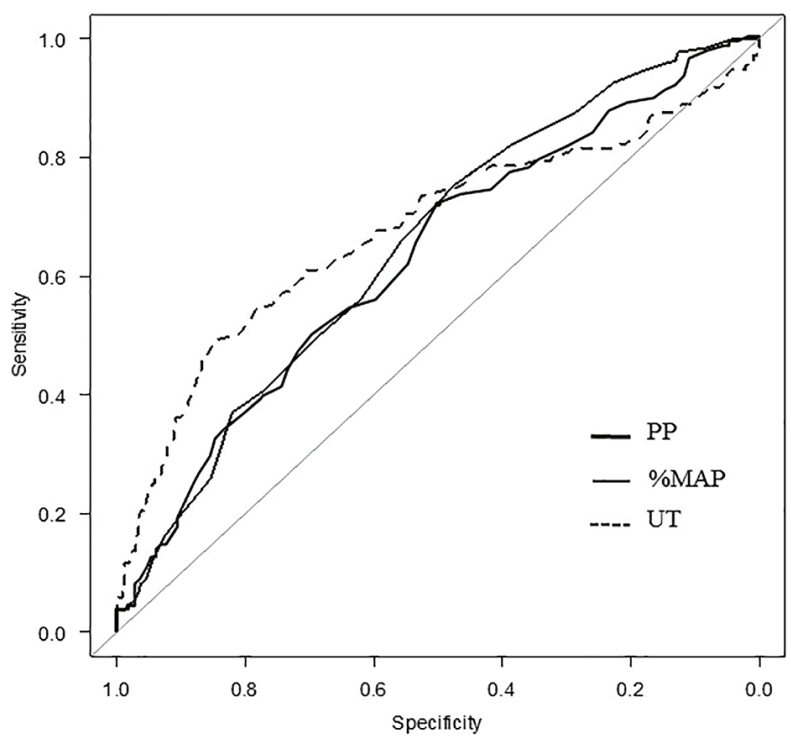

Figure 1. Receiver operating characteristic curve of pulse pressure (PP), \%mean atrial pressure (\%MAP) and upstroke time (UT) for detection of PAD. The mean area under the ROC curve and $95 \% \mathrm{Cl}$ for PP, $\%$ MAP and UT were 0.628 (0.569 - 0.687), 0.650 (0.593 - 0.706) and $0.676(0.615-0.737)$, respectively. And the cut-off level of PP, \%MAP and UT were $53.0 \mathrm{~mm} \mathrm{Hg}, 38.0 \%$ and $173.0 \mathrm{~ms}$, respectively.
0.039; mBP: OR 2.30, 95\% CI $1.22-4.37, \mathrm{P}=0.010 ; \mathrm{PP}: \mathrm{OR}$ $1.30,95 \%$ CI $0.69-2.46, \mathrm{P}=0.041)$.

\section{ROC curve for prediction of PAD}

Figure 1 shows ROC curves for the prediction of PAD. The ROC curves of significant parameters on multivariate analysis were prepared. The ROC curves show the fraction of true-positive results (sensitivity) and false-positive results (1 - sensitivity) for the cut-off levels. The cut-off levels that gave the maximal sensitivity and specificity for \%MAP and UT were $38.0 \%$ and $173.0 \mathrm{~ms}$ (\%MAP: sensitivity 0.473 , specificity 0.756 ; UT: sensitivity 0.846 , specificity 0.934 ), respectively. That for DBP and mBP, PP were $83.0 \mathrm{~mm} \mathrm{Hg}$ (sensitivity 0.558 , specificity 0.588 ) and $119.3 \mathrm{~mm} \mathrm{Hg}$ (sensitivity 0.838 , specificity 0.294), respectively (data not shown). The mean area under the ROC curve (AUC) and 95\% CI for \%MAP, UT, DBP and $\mathrm{mBP}$ were 0.650 (0.593 - 0.706), 0.676 (0.615 - 0.737), 0.554 $(0.483-0.604)$ and $0.543(0.481-0.606)$, respectively. Maximal AUC of BPs for the presence of PAD was PP. AUC, 95\% $\mathrm{CI}$ and the cut-off level for PP were 0.628 (0.569 - 0.687) and $53.0 \mathrm{~mm} \mathrm{Hg}$ (sensitivity 0.500 , specificity 0.721 ). AUC of PP was not different compared with that of \%MAP and UT (PP and \%MAP, $\mathrm{P}=0.534$; PP and $\mathrm{UT}, \mathrm{P}=0.184)$.

\section{Discussion}

\section{Cardiovascular risk factors and PAD}

Previous studies recommended that patients, who have cardio- 
vascular risk beyond 10\% with Framingham risk score (FRS), are administrated anti-platelet medications [12]. FRS was also considered a useful cardiovascular risk score in Japan [13]. FRS has the following six factors: (1 age; $(2$ gender; (3 HT; (4 DLP; (5 DM; and (6 smoker. In the present study, the presence or absence of cigarette smoking and past medical history could not be evaluated, because descriptions of these in medical records were insufficient. There were no differences in age and gender between the two groups. No significant difference was noted in the rate of lipid lowering medications. The Japan Atherosclerosis Society Guidelines recommend treatment paying attention to low-density lipoprotein cholesterol (LDL) [8]. Cardioprotective effect of LDL reduction by statin has also been reported [14]. The rate of medication with statin was significantly higher in the PAD group. No significant difference was noted in the antihypertensive medication administration ratio, but that of $\mathrm{CCB}$, an antihypertensive medication widely used in Japan, was significantly higher in the PAD group. The number of antihypertensive medications was also significantly higher in the PAD group, and SBP was also significantly higher in the PAD group. History of DM and the rate of glucose lowering medications were also significantly higher in the PAD group, showing that the PAD group had many cardiovascular risk factors.

\section{Pulse wave and PAD}

It is known that arterial stenosis elevates \%MAP and UT determined from pulse waves, and the normal levels of \%MAP and UT, indicating high pulse wave, are $<43-45 \%$ and $<160$ - $180 \mathrm{~ms}$, respectively [15]. In the present study, \%MAP and UT were significantly higher in the PAD group. Moreover, univariate and multivariate analyses indicate that \%MAP and UT were associated with prediction of PAD. AUCs of \%MAP and UT on ROC curves were also favorable. High pulse wave is a risk factor for stroke and cardiovascular disease [16]. Patients with PAD have low ABI. On the other hand, patients with peripheral artery calcification caused by DM and so on have high ABI. Thus, normal ABI has been decided into from 0.9 to 1.4 by guideline from the Japanese Circulation Society. However, previous study also indicated that $\mathrm{ABI}$ of 1.10 or less relates to subclinical atherosclerosis [17]. In the present study, the PAD patients with normal ABI were present. The \%MAP increase and UT prolongation appear in an early stage of atherosclerosis. Analysis of pulse wave and ABI are useful for these patients. It is known that the skin perfusion pressure (SPP) and toe brachial pressure index (TBI) are also useful in the diagnosis of PAD. In the present study, the majority of patients with PAD in the lower extremity ultrasonography directly underwent angiography. Therefore, SPP and TBI could not be analyzed.

\section{BPs between both groups}

SBP and PP were significantly higher in the PAD group, but DBP was lower in the PAD group, although the difference was not significant. Arteries constantly perfuse blood toward the periphery through the windkessel effect [18], but when arteriosclerosis progresses, vascular wall hardening decreases the windkessel effect, resulting in increases in SBP/PP and a decrease in DBP. The widest AUC on the ROC curve evaluated with BPs was PP, and this may have been due to the association between PP and arteriosclerotic disease in the present study. Association between PAD and PP has also been reported [19]. AUC of PP was not significantly different from AUCs of \%MAP and UT, suggesting that evaluation of PP is useful.

\section{Study limitations}

The first limitation is that this was a single-center retrospective study with a small number of patients. Choice of examinations as ABI and/or lower extremity ultrasonography was decided by attending physician because of retrospective study. The second limitation is that we evaluated PAD with lower extremity ultrasonography, not angiography. The blood flow velocity was evaluated in addition to the degree of stenosis on ultrasonography, but AHA guidelines recommend angiography as a PAD evaluation method. Evaluation of the internal iliac artery might be difficult in lower limbs echocardiography. We are planning to perform a study including angiography.

\section{Conclusions}

This small-scale retrospective study demonstrated that PP and pulse wave analysis with ABI report were associated with PAD in patients with normal ABI $(0.9-1.4)$. The measurement of PP and analysis of pulse wave could provide additional information for the diagnosis of PAD. In order to clarify the presence of PAD using ABI report, large-scale clinical studies are now required.

\section{Disclosure}

Takanori Ikeda received research funds and lecture fees from Mitsubishi Tanabe Pharma Co., Ltd, Daiichi-Sankyo, Co, Ltd., and Ono Pharmaceutical, Co, Ltd. The remaining authors declare that there are no conflicts of interest.

\section{References}

1. Ogura Y, Tsujita K, Shimomura H, Yamanaga K, Komura $\mathrm{N}$, Miyazaki T, Ishii M, et al. Clinical characteristics and intravascular ultrasound findings of culprit lesions in elderly patients with acute coronary syndrome. Heart Vessels. 2016;31(3):341-350.

2. Betge S, Kretzschmar D, Figulla HR, Lichtenauer M, Jung C. Predictive value of the augmentation index derived vascular age in patients with newly diagnosed atherosclerosis. Heart Vessels. 2016.

3. Alberts MJ, Bhatt DL, Mas JL, Ohman EM, Hirsch AT, Rother J, Salette G, et al. Three-year follow-up and 
event rates in the international REduction of Atherothrombosis for Continued Health Registry. Eur Heart J. 2009;30(19):2318-2326.

4. Diehm C, Allenberg JR, Pittrow D, Mahn M, Tepohl G, Haberl RL, Darius H, et al. Mortality and vascular morbidity in older adults with asymptomatic versus symptomatic peripheral artery disease. Circulation. 2009;120(21):2053-2061.

5. Guidelines for management of peripheral artery occlusive disease (JCS2009). Circ J. 2009;73(Suppl III):15071569.

6. Rooke TW, Hirsch AT, Misra S, Sidawy AN, Beckman JA, Findeiss LK, Golzarian J, et al. 2011 ACCF/AHA Focused Update of the Guideline for the Management of Patients With Peripheral Artery Disease (updating the 2005 guideline): a report of the American College of Cardiology Foundation/American Heart Association Task Force on Practice Guidelines. J Am Coll Cardiol. 2011;58(19):2020-2045.

7. Shimamoto K, Ando K, Fujita T, Hasebe N, Higaki J, Horiuchi $\mathrm{M}$, Imai $\mathrm{Y}$, et al. The Japanese Society of Hypertension Guidelines for the Management of Hypertension (JSH 2014). Hypertens Res. 2014;37(4):253-390.

8. Teramoto T, Sasaki J, Ishibashi S, Birou S, Daida H, Dohi S, Egusa G, et al. Executive summary of the Japan Atherosclerosis Society (JAS) guidelines for the diagnosis and prevention of atherosclerotic cardiovascular diseases in Japan -2012 version. J Atheroscler Thromb. 2013;20(6):517-523.

9. Shirai K, Utino J, Otsuka K, Takata M. A novel blood pressure-independent arterial wall stiffness parameter; cardio-ankle vascular index (CAVI). J Atheroscler Thromb. 2006;13(2):101-107.

10. Beneficial effect of carotid endarterectomy in symptomatic patients with high-grade carotid stenosis. N Engl J Med. 1991;325(7):445-453.

11. Kanda Y. Investigation of the freely available easy-to- use software 'EZR' for medical statistics. Bone Marrow Transplant. 2013;48(3):452-458.

12. Wilson PW, D'Agostino RB, Levy D, Belanger AM, Silbershatz H, Kannel WB. Prediction of coronary heart disease using risk factor categories. Circulation. 1998;97(18):1837-1847.

13. Suka M, Sugimori H, Yoshida K. Application of the updated Framingham risk score to Japanese men. Hypertens Res. 2001;24(6):685-689.

14. Shimomura M, Oyama J, Takeuchi M, Shibata Y, Yamamoto Y, Kawasaki T, Komoda H, et al. Acute effects of statin on reduction of angiopoietin-like 2 and glyceraldehyde-derived advanced glycation end-products levels in patients with acute myocardial infarction: a message from SAMIT (Statin for Acute Myocardial Infarction Trial). Heart Vessels. 2016;31(10):1583-1589.

15. Watanabe Y, Masaki H, Yunoki Y, Tabuchi A, Morita I, Mohri S, Tanemoto K. Ankle-Brachial Index, Toe-Brachial Index, and Pulse Volume Recording in Healthy Young Adults. Ann Vasc Dis. 2015;8(3):227-235.

16. Kawai T, Ohishi M, Onishi M, Ito N, Takeya Y, Oguro R, Takami Y, et al. Prognostic impact of regional arterial stiffness in hypertensive patients. Heart Vessels. 2015;30(3):338-346.

17. McDermott MM, Guralnik JM, Tian L, Liu K, Ferrucci L, Liao Y, Sharma L, et al. Associations of borderline and low normal ankle-brachial index values with functional decline at 5-year follow-up: the WALCS (Walking and Leg Circulation Study). J Am Coll Cardiol. 2009;53(12):1056-1062.

18. Reymond P, Westerhof N, Stergiopulos N. Systolic hypertension mechanisms: effect of global and local proximal aorta stiffening on pulse pressure. Ann Biomed Eng. 2012;40(3):742-749.

19. Korhonen P, Kautiainen H, Aarnio P. Pulse pressure and subclinical peripheral artery disease. J Hum Hypertens. 2014;28(4):242-245. 